\title{
Description de Ixodes brumpti n. sp. (Acariens, Ixodoidea) des Damans du Harrar (Ethiopie)
}

\author{
par P.-C. MOREL
}

\section{IXODES BRUMPTI n. sp.}

\section{Description.}

Holotype : 1 , Harrar, sur Heterohyrax brucei (1901, E. Brumpt, legit).

L'espèce est dénommée en hommage au professeur E. Brumpt qui l'avait récoltée.

\section{Femelle (fig. 1-2).}

CAPITUlum : basis capituli à bords latéraux convexes en vue dorsale ; cornes basidorsales moyennes en angle droit, modérément saillantes en raison de la continuité avec le bord postérieur légèrement convexe de la basis ; aires poreuses en largeur, triangulaires à bord antérieur concave, distantes entre elles de moins de leur largeur; socle hypostomal (antérieur au niveau de la base des palpes), en cône aussi long que large ; cornes basiventrales peu saillantes, arrondies, trapézoïdes; palpes six fois plus longs que larges (longueur dorsale : 0,7 mm) ; article palpal I à crête ventrale, sans pointe marquée ; collare à contour postérieur courbe ; hypostome à 1 rang basal de $1 / 1$ files de dents, puis 7-8 rangs de $2 / 2$ files, puis 4 rangs de $3 / 3$ files, puis $1-2$ rangs de $4 / 4$ files de dents, puis la coronule ; longueur de l'hypostome : $0,46 \mathrm{~mm}$; longueur capitulo-ventrale (de la pointe de l'hypostome au bord postérieur du collare) : 0,90 mm.

FACE DORSALE : scutum polygonal arrondi, un peu plus long que large ; longueur : $1 \mathrm{~mm}$; largeur : 0,80 $\mathrm{mm}$; sillons scapulaires et cervicaux complets; ponctuations moyennes en séries le long des sillons scapulaires et le long de petites rides transverses du champ cervical ; par ailleurs, ponctuations éparses moyennes et fines, irrégulièrement distribuées.

FACE VENTRALE : gonopore au niveau des coxae IV ; opercule ovale, à bord postérieur légèrement échancré ; sillon périanal en fer à cheval à branches très convergentes, 


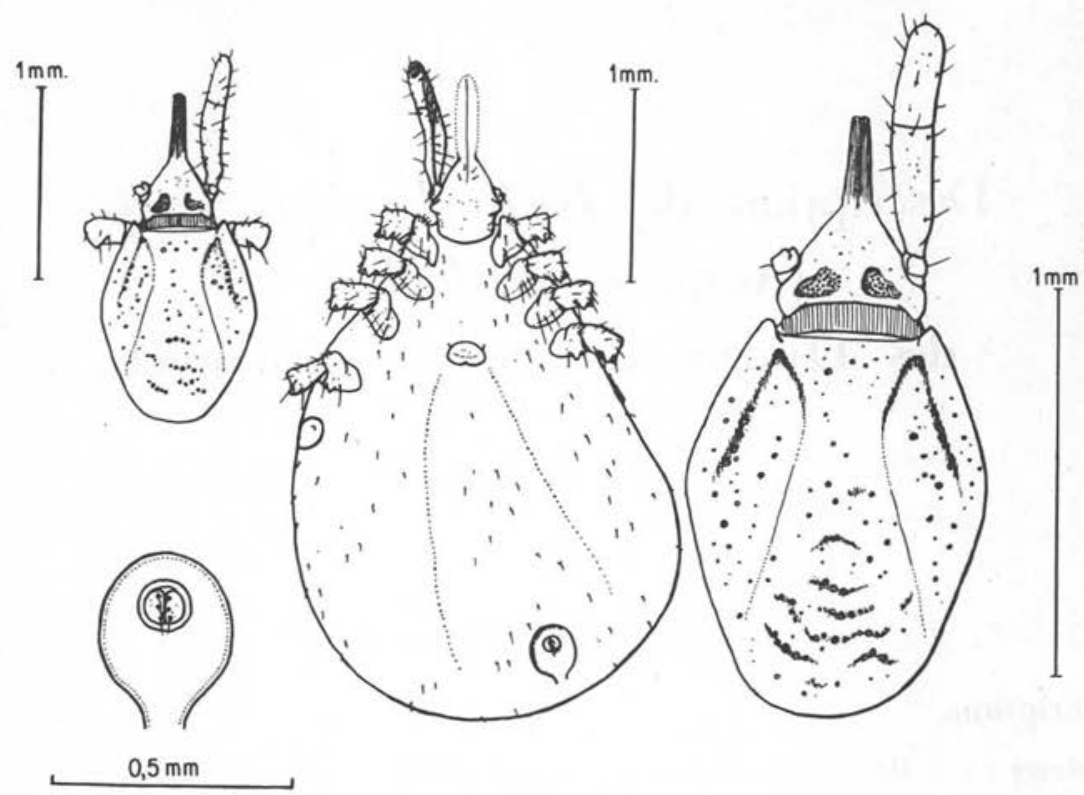

FIG. 1. - Ixodes brumpti, femelle; scutum et capitulum; face ventrale; détail du sillon préanal

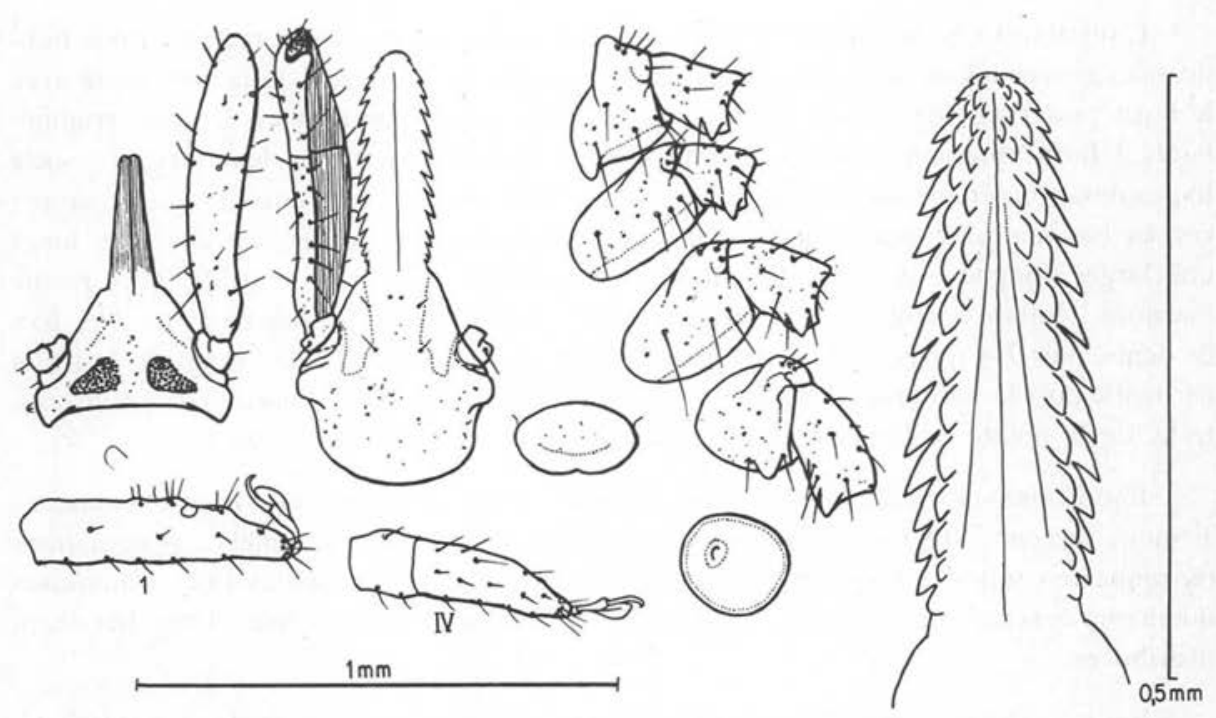

Fig. 2. - Ixodes brumpti, femelle; détail du capitulum en face dorsale et ventrale; détails des coxae, des tarses I et IV, du stigmate, de l'opercule gonoporal et de l'hypostome 
mais non fermé postérieurement ; sur le tégument, courtes soies effilées ; stigmates subcirculaires.

PATtes : syncoxa I à épine interne mousse, courte, sans épine externe, à subcoxa petite; processus coxal I anguleux; syncoxae II-III sans épines, à subcoxae minces à bord antérieur rectiligne, recouvrent moins du quart de la surface de la syncoxa ; coxa IV à épine externe moyenne ; trochanters à épine ventrale rétrograde en pointe ; tarses de proportions normales, sans gibbosité dorsale préterminale.

\section{Description.}

L'existence de trois paires de syncoxae et la forme en fer à cheval du sillon préanal placent 1 . brumpti dans le groupe de 1 . pilosus, également caractérisé par l'absence d'épines externes sur les coxae I (gr. I. elongatus, Bedford, 1929), ou I-II-III (gr. I. schillingsi, Neumann, 1901). I. brumpti appartient à cet ensemble d'Ixodes s.l. de la région éthiopienne, qui constitue certainement une branche autonome par rapport aux Ixodes $s$. str., dont le type est $I$. ricinus (L.).

Parmi les espèces du groupe de I. pilosus, Koch, 1844, dont les cornes basiventrales ne sont pas recourbées postérieurement, mais dont l'axe est approximativement perpendiculaire à la basis, 1 . brumpti se distingue par les épines ventrales rétrogrades des trochanters à la fois de $I$. pilosus et de $I$. oldi, Nuttall, 1913 ; ses cornes basiventrales sont d'ailleurs plus courtes que celles de ces deux espèces; les hypostomes sont de formules différentes ; chez $I$. oldi, les palpes sont ramassés relativement (trois fois et demie plus longs que larges); chez I. pilosus, également, l'allongement des palpes est moindre (quatre fois plus longs que larges); il manque d'ailleurs une redescription de 1 . pilosus suivant les critères actuels, ainsi que la description d'une espèce voisine d'Afrique orientale, dont la publication ne semble pas avoir paru (I. lewisi, Arthur : nomem nudum? in Arthur, 1958, 38).

Cet exemplaire de $I$. brumpti avait été vu par Nuttall et Warburton (1911: 233) et identifié comme I. ugandanus djaronensis, Neumann, 1907. Il s'agit pas du tout de cela : la redescription de $I$. djaronensis doit faire l'objet d'une de nos prochaines publications; ce dernier se situe également dans le groupe de $I$. pilosus, mais parmi les espèces à cornes basiventrales recourbées postérieurement en épines et représente peutêtre un synonyme de 1 . arabukiensis, Arthur, 1959.

On connaissait jusqu'à présent deux Ixodes parasites de damans: I. procaviae Arthur et Burrow, 1957 (du Kivu, Rwanda et Kenya, sur Procavia adolfi friederici et Dendrohyrax arboreus) et $I$. spinae Arthur, 1958 (du Transvaal, sur Procavia capensis) ; le premier appartient au groupe de 1 . rasus Neumann, 1899; le second au groupe de I. schillingsi.

Il convient donc d'ajouter à ces parasites de damans $I$. brumpti; dans la même région avait été d'ailleurs recueillie Haemaphysalis bequaerti Hoogstraal, 1956, sur Procavia brucei du Harrar ( $1 \delta^{*} 1 n$; V.01), déterminée autrefois par Nuttall et Warbur- 
ton (1915: 469), comme Haemaphysalis leachi small race. La femelle de 1 . brumpti et les $H$. bequaerti se trouvaient toujours dans la collection de E. Brumpt qui les avait récoltées (Institut de parasitologie, Faculté de médecine de Paris).

\section{Résumé}

Ixodes brumpti se place dans le groupe de 1 . pilosus à trois paires de syncoxae et sillon pré-anal en fer à cheval chez les femelles; il n'est connu que par un seul exemplaire et représente vraisemblablement un parasite habituellement associé aux damans d'Ethiopie.

\section{Bibliographie}

ARTHUR (D. A.), 1958, - New species of Ixodes ticks from eastern Africa, with description of the male of Ixodes oldi Nuttall, 1913. Parasit., 48 (1-2) : 38-69.

ARThUR D. A., 1959. - The Ixodes nairobiensis complex of species with description of four new species (Ixodoidea, Ixodidae). Rev. Zool. Bot. afr., 59 (1-2) : 137-157.

ARTHUR (D. A.) et Burrow (C.), 1957. - The Ixodes rasus group of African ticks with descriptions of four new species (Ixodoidea, Ixodidae). Bull. Mus. comp. Zool., 116 (9) : 493-537.

Hoogstraal (H.), 1956. - Notes on African Haemaphysalis ticks. III. The hyrax parasites $H$. bequaerti sp. nov., $H$. orientalis N. et W., 1915 (new combination) and $H$. cooleyi Bedford, 1929 (Ixodoidea, Ixodidae). J. Parasit., 42 (2) : 156-172.

Nuttall (G. H. F.) and Warburton (C.) 1911. - The genus Ixodes. Ticks, a monograph of the Ixodoidea, Cambridge (Univ. Press), 2 : I-XII, 105-348.

Nuttall (G. H. F.) and Warburton (C.), 1915. - The genus Haemaphysalis. Ticks, a monograph of the Ixodoidea, Cambridge (Univ. Press), $3: 349-550$.

Institut d'élevage et de médecine vétérinaire des pays tropicaux, Ecole vétérinaire, Alfort (Seine) Laboratoire national de recherches vétérinaires Georges-Curasson, Hann (Dakar) (Sénégal) 